Thomas Schwinn (Hrsg.)

Die Vielfalt

und Einheit

der Moderne

Kultur- und struktur-

vergleichende Analysen

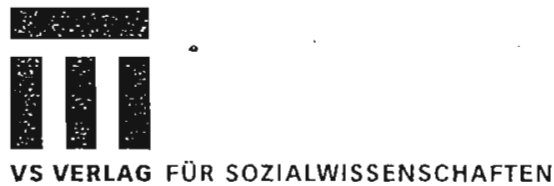




\section{Globalisierung und Modernisierung - Zentrale Annahmen der Globalisierungstheorien auf dem Prüfstand}

\section{Einleitung: Zur Perspektive der Globalisierungstheorien und dem Stand der Globalisierungsforschung}

Mit dem Stichwort der „Globalisierung“ ist in der Regel zumindest die Annahme angesprochen, daß sich gesellschaftliche Prozesse in zunehmenden Maße aus lokalen Zusammenhängen lösen (auch wenn Prozesse der lokalen Rückbindung parallel laufen) und dies kulturbedeutsame gesellschaftliche Auswirkungen zeitigt. Eine veränderte Form der Wahrnehmung von Territorialität (z.B. von der „Fläche“ zur „Erdkugel“, von der Nation zur Welt) wird in Verbindung gesetzt mit veränderten Formen der Wahrnehmung von Kausalität („Entkoppelung von Raum und Zeit“, „Kausalschleifen“) und gesellschaftlicher Strukturierung (vgl. dazu nur Schmidt 1999: 13). Die Ausweitung des Raumbezugs auf die Welt, so das Credo der Globalisierungstheorien, schafft früher oder später neue oder veränderte Formen von Sozialität und damit auch neue oder veränderte gesellschaftliche Problemlagen. Die meisten Globalisierungstheorien te ilen diese Annahme. Mehr noch: An ihr kann man im Regelfall erkennen, ob man es mit einer Globalisierungstheorie zu tun hat oder nicht. Auf der einen Seite ist es ihre starke Bezugnahme auf den Raum als Ausgangspunkt reflexiver Gesellschaftsstrukturierung, in der sie sich von Modernisierungstheorien unterscheiden. Auf der anderen Seite trennt sie die Prozeßperspektive der „Entgrenzung“, und damit die Annahme einer „NichtAbgeschlossenheit" der Globalisierung, von den (Welt)Systemtheorien. An solchen Unterschieden lassen sich die grundlegenden Annahmen der Globalisierungstheorien gut erkennen.

So gehen z.B. die Systemtheorie Luhmanns oder die Weltsystemtheorie Wallersteins (bei allen theoriearchitektonischen Unterschieden) gleichermaßen von einer ,Vollrealisation der Weltgesellschaft" (Luhmann) aus. Sie setzen Globalität als in Systemform durchgesetzt voraus und suchen von dort den Ausgangspunkt ihrer theoretischen Erklärungen. Es ist gerade deren Existenz als entgrenztes soziales System, so die Annahme, die dafur sorgt, daß regional dissipative Strukturen und selbstorganisierte Formen von Sozialität im regionalen Raum ent- und bestehen (vgl. Luhmann 1997: 808 f.; Wallerstein 1974, 1979). Globalisierungstheorien verzichten in der Regel auf diese Annahme und untersuchen eher die partielle, sukzessive Etablierung weltweiter Gesellschaftsformen (vgl. dazu auch Stichweh 2000: 14). Deswegen fügen sich ihre Ansätze bisher nicht unter eine gemeinsame systemtheoretische Architektur, sei sie kybernetischer oder marxistischer Provenienz. Der Blickwinkel eines gleichzeitig entsteh enden Systems einer höheren Systemebene, das Mechanismen der Globalisierung und der Lokalisierung als Mechanismen des eigenen Strukturaufbaus nutzt, ist zumindest 
kein bewußt gesetzter Ausgangspunkt für die Reflexionen der Globalisierungstheorien (vgl. Stichweh 2000: 14).

Im Vergleich zu den Modernisierungstheorien wählen die Globalisierungstheorien eine andere Leitunterscheidung, ein anderes Beobachtungsschema. Der Zeitbezug der Modernisierungstheorien, ihre Beobachtung nach dem Schema alt/neu, wird durch den Raumbezug ersetzt. Es werden Unterschiede in den "Terrains“ des Sozialen hervorgehoben. Die Analyse ist auf die „Überführungen“ von Lokalität in Globalität et vice versa konzentriert - ohne jedoch verkennen zu können, daß das Lokale nur im Globalen lokal ist und das Globale nur in der Form der Lokalisierung global wirklich wird. Mit dieser Umstellung des Beobachtungsschemas können Globalisierungstheorien (sofern sie auf bestimmte modernisierungstheoretische Annahmen verzichten) auf einige gravierende Schwierigkeiten der Theorien der Moderne reagieren. Nachdem diese ihr einfaches Modell einer Entwicklung vom Traditionalen zum Modernen aufgegeben haben, müssen sie heute die Gleichzeitigkeit von Moderne und Tradition als Moderne ausweisen (vgl. dazu u.a. Luhmann 1992). Thre Leitunterscheidung verliert damit an Instruktivität für ihre entwicklungstheoretischen Annahmen. Für viele Modernisierungstheorien ist es bis heute ein Problem, das sich gesellschaftlich neu Etablierende nicht als rationeller, besser oder fortgeschrittener auszuweisen und damit den Ballast älterer Evolutionstheorien über Bord zu werfen. Zwar gelingt es in den aufgeklärten Varianten die Steigerungen formaler Rationalität (in Anlehinung an Max Weber) als material irrational auszuweisen, aber nur selten die Annahme einer Steigerung formaler Rationalitäten im Zuge der Modernsierung zu streichen, also den Rationalisierungsvorbehalt aufzugeben.

Die Globalisierungstheorien können diese Schwierigkeiten theoretisch umgehen, indem sie anstelle des Zeit-Bezugs ${ }^{1}$ den Raum-Bezug ${ }^{2}$ in den Vordergrund stellen. Während mit Modernisierung auf der Gesellschaftsebene immer auch die globale Ausbreitung modernen Formen mitgedacht war, können es umgekehrt die Globalisierungstheorien gerade fragwürdig erscheinen lassen, ob mit der Globalisierung Modernisierung (als Steigerung formaler Rationalität) einhergeht. Denn dies erscheint keineswegs zwangsläufig so. Auch atavistische oder archaische Formen und Traditionen, traditionelle oder gar „rückschrittliche“ Methoden, Denkweisen und Organisationsformen können sich global in Gesellschaftsstrukturen übersetzen - man denke nur an die Vorstellungen einer „McDonaldisierung“ der Gesellschaft (vgl. Ritzer 2000). Und umgekehrt kann sich der lokale, regionale Raum auch ohne globale „Überführungen“ sehr weitgehend modernisieren. Anders gesagt, die Globalisierungstheorien können die Konvergenz- oder Divergenzfrage ohne Fortschrittsassoziation stellen, was umgekehrt den Modernisierungstheorien bis heute schwerfalltt. ${ }^{3}$ Darin liegt einer ihrer Vorteile. Sie können dadurch die Probleme der (älteren) Modernisierungstheorien vermeiden. Die Frage ist nur; ob sie dies in der Regel auch tun. Denn nicht selten scheinen sich hinter den Problematisierungen der Globalisierungstheorien modernisierungstheoretische Annahmen zu verbergen, scheinen diese zugleịch auch Modernisierungsmythen zu transportieren.

1 Als Zeit(/Raum)-Bezug.

2 Als Raum(/Zeit)-Bezug

3 Hierin liegt sicherlich auch ein Grund fur den zeitweisen Aufstieg der Theorien der Postmodeme.
Dieser Frage gehen die folgenden Ausführungen nach. Sie stellen einige zentrale Annahmen des Mainstreams der Globalisierungstheorien vor und hinterfragen sie auf ihr empirisches Fundament. Bewähren sich ihre Annahmen auf dem Prüfstand empirischer Forschung oder spiegeln sie nur mehr oder weniger eingängige Entwicklungs- und Modernisierungsmythen? Verschenken diese also mögliche Vorteile, die sich durch die Umstellung des Beobachtungsschemas ergeben, indem sie auf empirisch ungesicherte Entwicklungsannahmen und Fortschrittsassoziationen zurückfallen? Einen Beitrag zur Beantwortung dieser Fragen versuche ich im folgenden zu leisten, indem ich nacheinander auf die Rolle von Staaten (2.), von transnationalen Unternehmen (3.) und transnationalen Eliten (4.) sowie auf die Erosion sozialer Standards und kultureller Traditionen (5.) in einer sich globalisierenden Welt eingehe. Ich werde dabei sowohl den Bezug zum entwicklungssoziologischen Diskurs am Beispiel Ostasiens herstellen, um die Annahmen der Globalisierungstheorie einmal am Beispiel einer anderen Weltregion zu spiegeln, als auch neuere Befunde zu den Effekten der Globalisierung in Deutschland heranziehen. Ich werde mich hier insbesondere auf die Ergebnisse des Globalisierungsschwerpunkts der DFG beziehen ${ }^{4}$.

\section{Die Globalisierung und das Ende der Nationalstaaten}

Zu den wichtigsten und (ein)gängigsten Vorstellungen der Globalisierungstheorien gehört, daß viele Instrumente nationalstaatlicher Politik unwirksam werden und die nationale Souveränität im Zuge der Globalisierung erodiert (vgl. nur Albrow 1998: 419). Die Globalisierungsdiskussion kulminiert u.a. in der Annahme, daß die alte Form des Nationalstaats, die nationalstaatliche Verfaßtheit von Wirtschaft und Gesellschaft keine entscheidende Rolle mehr spielt und von neuen, globalen Formen der Politik abgelöst wird (vgl. z.B. Camilleri/Falk 1992; Reich 1993; Narr/Schubert 1994; Ohmae 1995). So sprechen z.B. Narr/Schubert von einer Denationalisierung der Ökonomie (vgl. Narr/ Schubert 1994: 28 ff.) und für Ohmae haben Nationalstaaten als Grundeinheiten der globalen Wirtschaft ausgedient (vgl. Ohmae 1995: 26). Dafür zeichnet nach Ohmae eine Industriedynamik verantwortlich, die sich längst aller nationaler Kontrollen entledigt hat. Die Gelder, die heute über Ländergrenzen flössen, seien zumeist in Privatbesitz. Auf keiner der beiden Seiten müsse mehr eine Regierung beteiligt sein. Die Industrie sei global orientiert, ihre Ansiedlungspolitik immer weniger von Regierungsentscheidungen abhängig (vgl. ebd.: $15 \mathrm{f}$., $115 \mathrm{f}$.).

In dieser Vorstellung einer Erosion nationalstaatlicher Regulierung kontrastiert die Globalisierungstheorie eigentümlich mit dem entwicklungssoziologischen Diskurs. Hier gehören sog. neomerkantilistische Ansätze zum festen Repertoire der Erklärung von 4 Dabei erfahren folgende Projekte, bzw. Projektzusammenfassungen besondere Aufmerksamkeit: Rolf G. Heinze, Heiner Minssen: Regionalisierungstendenzen in Wirtschaft und Politik am Beispiel der Umdistribte als ind Opel in Bochum und Opel in Eisenach; Jurgen Feldhoff, Philipp Hessinger: Industriedistrikte als industriepolitisches Handlungsfeld, Hermann Biehler, Joachim Genosko et al.: Region ale Netzwerke und regienalor Arbeilsmarkt. Kumulative Prozesse zirkularer Verursachung?, Ulrich Mückenberger: Zivilgesellschaft und disperse Interessen, Stephan Leibfried: Globalisierung und Sozialhilfere- 
Entwicklung. Am Beispiel der Entwicklung in Ostasien läßt sich dies gut erläutern. In der Analyse der Wirkkraft der nationalstaatlichen Regulierung in den ostasiatischen Ländern sind sich viele sozialwissenschaftliche Autoren einig darin, daß ,market explanations", wie sie z.B. Porter (1990), Weede (1990) oder Ohmae (1995) formulieren, deren Entwicklung nicht gerecht werden (vgl. z.B. Amsden 1989; 1990; Wade 1990; Henderson 1993). Weder können sie die Entwicklung noch die Unterschiede in der Entwicklung der ostasiatischen Länder erklären (vgl. Hamilton/Biggart 1988: 64 ff.) In fast allen ostasiatischen Ländern (mit Ausnahme Hongkongs) hatte der Staat Formen korporatistischer Regulierung angewandt, um die Industrialisierung zu fördern. Es entstanden „Entwicklungsstaaten"s, die mittels hoch qualifizierter und machtvoller staatlicher Verwaltungsapparate für eine zentrale staatliche Steuerung sorgte, die jedoch halt machte, wenn es um die Aufrechterhaltung des Marktes und den Schutz des Privateigentums geht. Ihnen gegenüber blieben die Staatsinterventionen streng limitiert. Dabei stellte der autoritäre Korporatismus - mit seiner abgeschwächten Variante in Japan ein zentrales Element im System der regulierten Märkte (,governed market") und ein Grund für ihren ökonomischen Erfolg dar (vgl. Wade 1990: 26 ff.). Mittels Landverteilung, Kontrolle des Finanzsystems, ökonomischen Stabilitäts- und Schutzpolitiken, Exportorientierung, Technologietransfer- und selektiver Industriepolitik hatte nach Wade der Staat den Markt in den ostasiatischen Ländern regiert. Hinzu kamen „repressive labor-systems", die (wenn auch in unterschiedlicher Weise) ein wichtiges Element des autoritären Korporatismus in Ostasien waren (Castells 1992: 176 ff.; vgl. dazu auch Deyo 1989).

Am Beispiel Ostasiens mit seinem außerordentlich hohen Maß an weltwirtschaftlicher Integration ließ sich also klar erkennen, daß eine zunehmende "Globalisierung“ der Wirtschaft nicht zwangsläufig, wie von vielen Globalisierungstheoretikern vermutet, mit der Erosion traditioneller Instrumente staatlicher Politik oder gar ihrem Obsolet-Werden korreliert sein muß. Im Gegenteil verdankte sich der Bedeutungszuwachs staatlicher Politiken in Ostasien der erfolgreichen Integration in globale Märkte; so wie diese wiederum starke Entwicklungsstaaten zur Voraussetzung hatte.

Doch manchen erschien dies nur als eine Phase "nachholender Modernisierung". Die ostasiatischen Entwicklungsländer durchliefen diese im Nachholen der „Erste Moderne" mehr oder weniger zwangsläufig, um dann, in späteren Phasen, in das Fahrwasser westlicher Gesellschaften zu münden und im Zuge voranschreitender Differenzierung ebenso wie diese mit der schwindenden Bedeutung der Staaten im Zeitalter der Globalisierung zu kämpfen zu haben. Und die seit Mitte der 80er Jahre sich vollziehende Entwicklung zu sog. Post-Entwicklungsstaaten ${ }^{6}$, die sich in vielen ostasiatischen Ländern abzeichnet, schien ihnen recht zu geben. Die dritte Welle der Demokratisierung traf

5 Das Konzept geht in seinen Ursprüngen auf Hirschman (1958) und Gerschenkron (1962/69) zuruck und wurde von Johnson (1982) am Beispiel Japans ausgearbeite

$6 \mathrm{Zu}$ seinen Charakteristika gehoren ein unter dem Aspekt des industriepolitischen Dirigismus zurückgenommener Staat, eine stärkere Teilung der institutionellen Macht und Kräfte, eine viel mehr als fruher auf Koordinationsfunktionen zuruckgezogene Verwaltung, die relative Autonomie der Kapitalinteressen und die Lockerung der ehemals straff festgezurten korporatistischen Arrangements (vgl. dazu auch Hahm/ Plein 1997).
Ostasien mit einiger Wucht, machte dem politischen Autoritarismus den Garaus und ließ Zweifel an der Gestaltungsmacht der vormals ,starken Staaten“ aufkommen

Doch ein genaues Hinsehen bringt hier Ernüchterung. Während und nach den neoliberalen „Schocks“ gewannen viele Staaten in Ostasien wieder deutlich an Terrain und fuihrten die Restrukturierung der ostasiatischen Industrielandschaften zwar in modifizierter Weise, aber nichtsdestotrotz mittels staatlicher Regulierungsmaßnahmen durch. Südkorea ist dafür ein gutes Beispiel

So höhlte die Liberalisierungspolitik in Südkorea, die mit dem Ziel des OECDBeitritts in den 90er Jahren konsequenter umgesetzt wurde, die alten korporatistischen Kontrollstrukturen zwischen Staat, Banken und den südkoreanischen Großunternehmensgruppen (Chaebol) zunächst aus. Der liberal stärker zurückgezogene Post-Entwicklungsstaat setzte den expansiven, mit globalem Kapital finanzierten Unternehmenspolitiken nichts entgegen. Er war daher mit verantwortlich für Südkoreas ökonomischen Einbruch während der asiatischen Finanzkrise. In der Restrukturierung nach de Krise spielten ebenfalls neoliberale Anstrengungen zunächst eine große Rolle (HartLandsberg/Burkett 200 1: 414). ${ }^{7}$ Die Liberalisierungsmaßnahmen wurden aber von einem gar nicht liberal agierenden Staat mit großer Effektivität umgesetzt. Der Staat besann sich seiner alten Mittel, z.B. des Zugriffs auf die Kreditpolitik, nun aber, um das Chaebolsystem aufzubrechen. Das alte Wirtschaftsmodell, das in den 90er Jahren bereits in Erosion begriffen war, wurde mit Beginn des neuen Jahrzehnts zwar mit Paukenschlägen politisch verabschiedet. Aber seine Desorganisation wurde in staatlich handgreiflicher Weise vorangetrieben. Der in den 90er Jahre liberal zurückgezogene Staat war zurück und führte nach der Krise eine beispiellose „Modernisierung von oben" durch

Zwar gibt es auch in Ostasien hinreichende Anzeichen für nationalstaatliche Deregulierungsimpulse, aber diese weisen zunächst nur auf die zunehmende Bedeutung von zwei weiteren Regulierungsebenen hin: der regionalen und globalen Regulation. Die Bedeutung nationalstaatlicher Regulierung mag vor diesem Hintergrund relativiert werden, aber von globalen Erosionserscheinungen kann keine Rede sein. So wird in der Entwicklungssoziologie gegenüber markt- und handelszentrierten Interpretationen vielfach nachgewiesen, wie sehr die Nationalstaaten weltweit entwicklungsfördernd oder -hemmend in die wirtschaftliche Entwicklung eingreifen (vgl. nur Amsden 1989; Cumings 1989; 1997; Wade 1990; Henderson/Appelbaum 1992 u.v.a.). In besonderer Weise zeigten dies auch ökonomische Spätentwickler wie Deutschland und Japan oder eben neuerdings die asiatischen Schwellenländer (mit Ausnahme Hongkongs). Sie machten deutlich, daß im Zuge zunehmender weltwirtschaftlicher Integration auch di e Dominanz nationalstaatlicher Regulierung zunehmen kann (vgl. dazu Hirschman 1958; Gerschenkron 1962; Johnson 1982). Auch China wird auf absehbare Zeit durch einen 7 Sudkoreas Wirtschaft offnete sich in der Folge sehr weitgehend für ausländische Konkurrenz und ausländische Investitionen (vgl. auch Business Week, 10.6.2002). Der Geldmarkt wurde Ende 1998 vollkomfeindliche wurden ermoglicht. Die Übernahmen von inlandischen Firmen durch auslundische Investofendiche, wurden ermoglicht. Die Ubenahmen von inlandischen Firmen durch auslandische lnvestoBurk 2001: 415) und den Low jahren historische Rekordhohen (allerdings von einem sehr niedrigen Ausgangspunkt aus). 
außerordentlich starken Einfluß zentralstaatlicher Lenkung geprägt sein, dies heißt, daß weniger, China' als Gesellschaft und Wirtschaft, sondern der sozialistische Staat (auch: die Partei) als globaler Akteur, sichtbar' werden wird.

Staaten sind-das zeigen die empirischen Analysen entgegen mancher Annahmen der Globalisierungstheorie sehr deutlich - nicht nur in Ostasien nach wie vor entscheidende gesellschaftliche und weltwirtschaftliche Akteure. Sie prägen in ihren Territorien Lebens- und Arbeitsbedingungen in nachhaltiger Weise. Es soll hier nicht geleugnet werden, daß es im Zuge der Globalisierung Veränderungen in bezug auf die nationalstaatliche Souveränität gegeben hat. Aber keineswegs können wir derzeit davon ausgehen, daß die global agierende Wirtschaft (wie von vielen Globalisierungsdenkern behauptet) die Grundlagen der Nationalstaaten generell untergräbt (vgl. Beck 1997: 14) und deren Ende heraufbeschwört. Dies scheint eher einer der eingängigen (Modernisierungs-)Mythen der Globalisierungstheorien zu sein.

Eine Erklärung für diese nachhaltige Präsenz der Nationalstaaten bieten sowohl Systemtheorien marxistischer als auch kybernetischer Provenienz an. In der Perspektive der Kapitalismustheorie marxistischer Provenienz verallgemeinert der kapitalistische Weltmarkt - will man Mandel (1972) folgen - wohl die kapitalistische Warenzirkulation, nicht aber die kapitalistische Warenproduktion. ${ }^{8}$ Kein , homogenes kapitalistische Milieu" entsteht, sondern das kapitalistische Weltsystem verstärkt gerade eine „Vielfalt und Unterschiedlichkeit gesellschaftlicher Entwicklungen“, die weit über die Divergenz zwischen Entwicklungs- und Industrieländer hinausgeht (vgl. ebd.: $78 \mathrm{ff}$.; siehe zu dieser Auslegung von Mandel auch Trimberger 1979: 129). Das Hervorbringen der nationalen und regionalen Vielfalt der kapitalistischen Warenproduktion erscheint dann als funktional; es begründet erst die zahlreichen strukturellen Variationen im weltweiten Fluß der Kapitalströme. Auch die kybernetisch inspirierte Systemtheorie macht dies auf Basis eines ganz anderen Theorierahmens deutlich. Bei der Kopplung von Politik und Wirtschaft spielen nach Luhmann staatlich erhobene Steuern, Abgaben, nationalstaatliche Konditionierungen von Profitchancen durch Infrastrukturen der Ressourcenallokation nach wie vor eine hervorgehobene Rolle (vgl. dazu auch Luhmann 1997: 781). Insgesamt kann man sagen: Je mehr Kapital fluktuiert und nationale Räume überschreitet, je kontingenter die Produktion gegenüber tradierten nationalen Bindungen wird, desto sensibler und flexibler reagieren Kapital und Anlagevermögen auf national, regional und lokal konditionierte Infrastrukturen der Ressourcenallokation. Mit dieser Sensibilität der verselbständigen Geldwirtschaft und der daran angeschlossenen Kapitalakkumulation für differierende lokale und nationale infrastrukturelle Bedingungen reproduziert sich die Bedeutung von staatlichen Einflußnahmen in einer globalisierten Ökonomie. Nationalstaaten konditionieren den Fluß der Kapitalströme und geben so der Weltwirtschaft u.a. ihre Struktur. Im Zuge der Globalisierung kommt es daher auch zur Aufwertung nationalstaatlicher Politiken, da sie mit den Profitchancen (nach Maßgabe der Verfügbarkeit von Ressourcen) auch den Fluß von Ressourcen selbst, also zum Beispiel von Kapital und Arbeit, mitbestimmen können.

8 Mandel weiter: „Noch abstrakter ausgedruckt: Die Erscheinungen des Imperialismus sind durch eine fehlende Homogenisierung der kapitalistischen Weltwirtschaft żu erklären" (Mandel 1972: 78; Hervorhebung im Original).
Der Effekt der Globalisierung kann deswegen in der Perspektive der Systemtheorien nicht in einer weitgehenden Aufgabe nationalstaatlicher Regulation oder Konditionierung von Ökonomien gesehen werden. Vielmehr dränge sich die segmentäre Differenzierung des weltpolitischen Systems nach Luhmann nun allen Territorien auf: „Es gibt keine Gebiete, die an Politik teilnehmen (und es gibt auch keine Gebiete, die das vermeiden können); ohne daß sie die Form von 'souveränen' Staaten annehmen (Luhmann 1998: 379).

\section{Globale Entwicklung und transnationale Unternehmen}

Während die Nationalstaaten für die Globalisierungstheorien als globale Akteure an Bedeutung verloren haben, erfahren die global agierenden Unternehmen in ihren Vorstellungen und Annahmen eine enorme Bedeutungsaufwertung. Sie figurieren in den Globalisierungstheorien als Träger und Ausdruck einer neuen Form von Globalisierung und erscheinen zugleich als einflußreiche Träger einer Modernisierung insbesondere zurückgebliebener Wirtschaften. Mit ihnen wird eine Beschleunigung der Globalisierung mit entsprechenden Prosperitätschancen ebenso verbunden wie eine weitergehende Erosion sozialer und kultureller Standards. Rekordgewinne und der gleichzeitige massenhafte Abbau von Arbeitsplätzen, Verlagerungen der Produktion und ein massive Flucht aus dem nationalstaatlichen Steuerzugriff werden auf der einen Seite angeprangert (vgl. dazu Beck 1997: 19-22). Auf der anderen Seite werden wundersame Prosperitätseffekte propagiert, die sich einstellten, wenn man den transnationalen Unternehmen nur die Tür öffne und eine möglichst freie Beweglichkeit des Kapitals im eigenen Land garantieren könne. Diese stellten sich umgekehrt auch dann ein, wenn die weltwirtschaftliche Integration von regional angesiedelten und entsprechend vernetzten Unternehmen vorangetrieben werde.

Im weltweiten entwicklungssoziologischen Diskurs ist hier der Fingerzeig auf die ostasiatischen (Schwellen)Länder üblich, die als Prototypen für diesen Zusammenhang stehen. Ihr rasanter Aufstieg zu Wachstum und Prosperität wurde zum einen den positiven Effekten der Weltmarktintegration ihrer Unternehmen zugerechnet. Zum anderen gelten die Wirtschaften Asiens, allen voran die vier kleinen Tiger, den Anhänger der neoliberalen Theorie auch in der Frage der positiven Aspekte einer Ansiedelung global agierender Unternehmen als ausgezeichnete Beispiele, um regionale Vorteile der Ansiedelung zu belegen. Nicht zuletzt hat sich in den letzten Jahrzehnten die Politik des IWF in dieser Weltregion an der These der positiven Effekte orientiert. So trieb diese während der Asienkrise die weitere Öffnung der ostasiatischen Wirtschaften mit Erfolg voran. Der Einzug der internationalen Konzerne in Südkorea und die Etablierung zahlreicher strategischer Allianzen mit inländischen Unternehmensgruppen stehen für diese Entwicklung. Sie bestätigte für viele Beobachter, daß man mit dieser Politik richtig lag. Denn Südkorea und einige andere asiatischen Länder schafften den Weg aus der Krise in atemberaubender Geschwindigkeit (vgl. dazu u.a. Pohlmann 2002, 2004). Die weltweit freie Beweglichkeit von Kapital und Unternehmen, von der sich die neoliberale Theorie so viel verspricht, schien in diesen Fällen ihre Versprechen einzulösen. 

worh auch hier weist eine genauere Untersuchung des Zusammenhangs auf die Frag-
.

Das Besondere an der Entwicklung der ostasiatischen Ökonomien ist gerade im nas - die Ansied beiden Stadtstaaten und neuerdings Chihaben ihre wirtschaftlichen Erfolge Unternehmen so gut wie keine Rolle spielte. Sie größerem Umfange erreicht haben. gerade ohne ausländische Direktinvestitionen in von Japan, Sijdkorea und haben. Dies gehört zu den gemeinsamen Besonderheiten Jahren belegten Südkorea und Japan in der zunehmenden Liberalisienung in den $90 \mathrm{er}$ 1992 bis 1997 hat Surdkorea nur eine in dieser Frage weltweit die letzten Plätze. Von Kapitals zur nation zweitletzten Platz in der Welt belegt (ang von $0,9 \%$ erreicht und damit vor Japan den pur die Liste mit $25.4 \%$. Welt belegt (vgl. Korea Herald, 20.8.1998). Während̈ Singa( Südkorea ab. Tab. 1: Beitragsrate der auslandischen Direktinvestitionen zur Bruttokapitalbildung in unterschiedlichen
asiatischen Landem 1970-1997

\begin{tabular}{|c|c|c|c|}
\hline Iand/ & & & $\varnothing-\%$ \\
\hline Singapur & $1971-80$ & $1981-90$ & $1992-97$ \\
\hline Singapur & $15,8 \%$ & $26,2 \%$ & $25,4 \%$ \\
\hline 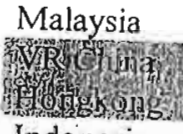 & $13,6 \%$ & $11,3 \%$ & $18,1 \%$ \\
\hline $\begin{array}{l}\text { Indonesien } \\
\text { ond }\end{array}$ & $\begin{array}{l}3,5 \% \\
30\end{array}$ & $1,5 \%$ & $4,6 \%$ \\
\hline Thailand & $2,3 \%$ & $4,8 \%$ & $5.0 \%$ \\
\hline 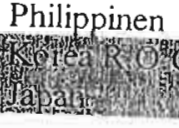 & $1,0 \%$ & $\begin{array}{l}3,8 \% \\
0,8 \%\end{array}$ & $4,6 \%$ \\
\hline
\end{tabular}

Quelle: Jwa/Huh 1998: 11; UNCTAD Data Base

Im Vergleich der (ost-)asiatischen Ökonomien läßt sich also ein solcher Zusammenhang zwischen der Ansiedlung globaler Unternehmen und der Entwicklung zur wirtchaftlichen Prosperität nicht einfach konstatieren. Vi und der Entwicklung zur wirtsten ostasiatischen Ökonomien in den vergangenen. Vielmehr haben die prosperierendche Ansiedlung ,verzichtet" Erst mit Chingenen vierzig Jahren bewußt auf eine soletwas zu wandeln.

Aber auch in Deutschland bringen die empirischen Befunde des Globalisierungs diskurs. Eine genauēre Untersuchung transnationaler Unternehmen und der von Zusammenhangs zwischen der Ansiedlung differenzierte Befunde anen und der von diesen ausgehenden Prosperitätseffekte bring der Globalisierungstheorie sprechen.
Die Untersuchungen von Heinze/Minssen (2001) und Feldhoff/Hessinger (2001) mahnen auf Basis der Untersuchung von Ansiedlungspolitiken in deutschen Regionen zu einer Skepsis, die ebenso im kritischen entwicklungssoziologischen Diskurs zu finden ist. Beide zeigen an den von ihnen untersuchten Fällen auf, daß mit der Ansiedlung von multi- oder transnationalen Unternehmen in Deutschland keine oder sogar negative Auswirkungen für die Entwicklung von Regionen einhergingen. Ihre empirischen Ergebnisse machen darauf aufmerksam, wie voraussetzungsvoll die Verwirklichung positiver Effekte ist und im Zweifel eher die Gegenthese stark.

In der Frage der Beschaffung sowie in der Frage der Qualifizierung des Personals erwies sich nach den Ergebnissen von Heinze/Minssen der "global player" Opel in seinen strategischen Entscheidungen als weitgehend unabhängig von regionalen Bezügen. Und in bezug auf die Infrastruktur in der Region bestand kein Handlungsbedarf. Da beide untersuchten Werke, das in Bochum und insbesondere das in Eisenach, Standorte des ,global player" mit wenigen oder, wie im Falle Eisenachs, keinen wesentlichen Kernfunktionen waren, waren die Chancen fur die Herausbildung regionaler Netzwerke und positiver Ausstrahlungseffekte für die Region nach Heinze/Minssen eher schlecht. Die Entstehung von Netzwerken und ihre positiven regionalökonomischen Effekte seien daher, so Heinze/Minssens These, keineswegs selbstverständlich und nicht nur in Deutschland an die Bedingungen der Ansiedlung für "global player" geknüpft. Auch die Arbeiten von Feldhoff und Hessinger weisen am Beispiel der "global player" in der Schiffbauindustrie in Rostock auf denselben Sachverhalt hin. Sie schreiben: „In einem Fall waren die Auswirkungen dieser Positionskämpfe (in globalen Warenketten) auf die regionalen Kontexte desaströs, in anderem Fall zumindest äußerst ambivalent" (Feldhoff/Hessinger 2001: 6). Dabei geben sie als Bedingungen fur diese Unterschiede auf der einen Seite den Grad der Standardisierung von Tătigkeiten, die Art der Ressourcen in Wertschöpfungsketten und die jeweilige Gestaltung und Innovativität der Umwelten an. Auf der anderen Seite spielten aber auch Prozesse der Verstärkung der jeweiligen Ausgangsbedingungen eine Rolle, also Abwärtsspiralen ebenso wie Aufstiegsidynamiken.

Insgesamt bringen diese und andere Globalisierungsprojekte des DFG-Schwerpunktes Klarheit und Emüchterung in einen Diskurs, der nicht selten von einem „Kurzschluß" von der bloßen Anzahl global orientierter und globaler Unternehmen in einer Region auf die Prosperitätschancen geprägt ist. Diese Ernüchterung kann helfen, den Globalisierungsdiskurs in dieser Hinsicht empirischer zu führen und ihn von den politischen Spielwiesen neo-liberaler Weltanschauungen auf das wissenschaftliche Feld fundierter empirischer Forschung zurickzuholen.

Dies gilt auch fur die Frage der Vorteile der weltwirtschaftlicher Integration re gionaler Unternehmen. So zeigen Genosko/Biehler u.a. zwar fur die Medienwirtscha ft in München auf, daß bei dieser durch die Integration regionaler Unternehmen in den Weltmarkt positive Effekte zum Tragen kamen und zu starken lokalen Standortvorteilen fuhrten. Sie weisen aber auch darauf hin - und sorgen damit für eine erste Relativierung dieser Annahme - daß dies keineswegs generell fur alle Industrien im Munchner Großraum gilt. So haben sich z.B. aus dem weltweit orientierten Münchner Fahrzeugbaus heraus - ganz entgegen der neoliberalen Annahme im Globalisierungsdiskurs - 
weder enge regionale Kooperationsnetzwerke entwickelt noch sich regionale Synergien mit positiven Effekten für den regionalen Arbeitsmarkt ergeben. Zwar sei der Fahrzeugbau für die Standortbildung wichtig und seine quantitative Bedeutung hoch, aber eine regionale Einbettung durch Kooperationen und Netzwerke, die sowohl die Netzwerke als auch die Region voranbringe, gebe es nicht. Auch die differenzierten Befunde der Untersuchungen von Heinze/Minssen und Feldhoff/Hessinger gemahnen daran, diese These nicht einfach und plakativ zu übernehmen und die Wirkkraft dieses $\mathrm{Zu}$ sammenhangs zu überschätzen. Die Untersuchung unterschiedlicher Industriedistrikte und Regionen (darunter Tuttlingen, Rostock, Bochum und Eisenach) in Deutschland zeigt, daß der Zusammenhang zwischen globaler Integration regionaler Unternehmen bzw. ihrer Netze und regionaler Entwicklung zur Prosperität kein zwangsläufiger ist und sehr von den regionalen Bedingungen abhängt, unter denen die weltwirtschaftliche Integration stattfindet.

Die Ergebnisse des Globalisienungsschwerpunktes weisen darauf hin, daß es für die Untersuchung von Globalisierungsprozessen zunehmend wichtig ist, Bedingungen herauszufinden und zu systematisieren, unter welchen positive Effekte der globalen Integration fir die regionale Entwicklung entstehen und unter welchen nicht. Hier steht die Globalisierungsforschung derzeit noch am Anfang. Von den generellen Annahmen der Globalisierungstheorie jedenfalls muß sie sich um so mehr verabschieden, als diese eher (Modernisierungs-)Mythen des neoliberalen Diskurses reflektieren als den Stand der bereits verfulgbaren Forschung.

\section{Die Entstehung globaler Wirtschaftseliten}

Mit der ,schicksalhaften“ Bedeutung der transnationalen Unternehmen wurde im Globalisierungsdiskurs auch die Entstehung eines transnationalen Managements, einer neuen „Weltklasse\% des Managements hervorgehoben. Ähnlich wie in der älteren Modernisierungstheorie fungierten diese' in der Globalisierungsliteratur als Wegbereiter moderner, global orientierter Denkweisen und Formen des Wirtschaftens. Moss Kanter sprich hier sogar von einer neuen „Weltkultur des Managements“ und der Herausbildung einer globalen Elite (Kanter 1997: $93 \mathrm{ff} ., \mathrm{vgl}$. dazu Hartmann/Kopp 2001)

Aber auch hier weisen die Ergebnisse unterschiedlicher Studien zum internationalen Management in eine andere Richtung. Zwar ist z.B. fur viele grenzilberschreitende Fusionen symptomatisch, das haben DaimlerChrysler, BMW/Rover und andere ge zeigt, daß zunächst von allen Beteiligten auf die Internationalität des Unternehmens und seiner Spitzenmanager hingewiesen wird, doch schon nach relativ kurzer Zeit zeigt sich in der Regel ein ganz anderes Bild: Der neue Konzern und vor allem das Topma nagement weisen eine eindeutig nationale Prüung auf (vgl daz Auch heute noch erweist sich in Europa, USA und Asien selbst bei den größten Unternehmen das Topmanagement uberraschenderweise als gering intemationalisiert oder international erfahren. Nur 2\% bis 7\% des Topmanagements der 100 grổ nehmen in Deutschland, Großbritannien, Frankreich und den USA sind Ausländer und nur $7 \%$ bis $16 \%$ haben lăngere Auslandsaufenthalte zu verzeichnen (vgl. Hartmann
2002). Dies gilt erstaunlicherweise auch für die jüngeren Jahrgänge des Topmanagements (vgl. ebd.). Das heißt: Vor der sehr starken Internationalisierung der Untemehmen bleibt jene des Topmanagements in Deutschland und den wichtigen westlichen Industrieländem zurück.

Auch die Vorstellung, daß das moderne Management sich als "Globetrotter" zwischen den trans- und multinationalen Unternehmen bewege, gleichsam zwischen diesen zirkuliere und so die globale Modeme verbreite, hält der Empirie nicht stand. [n Deutschland sind nach Windolfs Untersuchungen rund $40 \%$ der zentralen Figuren der Wirtschaft - Vorstandsvorsitzende großer Unternehmen oder Banken, die mehrere Aufsichtsratsposten wahmehmen („,big linkers“) - länger als 10 Jahre im Unternehmen. In den USA, das gerne als Vorreiter dieser globalen Entwicklung verstanden wird, liegt dieser Anteil sogar bei fast 50\%.

Tab. 2: Durchschnittliche Betriebszugehorigkeit der „Big Linkers“ in Deutschland, GroBbritannien und den USA 1999

\begin{tabular}{|l|l|l|l|}
\hline Jahre & Deutschland & Großbritannien & USA \\
\hline $\mathrm{N}$ & 169 & 116 & 766 \\
\hline & & & \\
\hline 0 & $25,3 \%$ & $37,9 \%$ & $25,8 \%$ \\
\hline $1-5$ & $16,6 \%$ & $19,0 \%$ & $15,2 \%$ \\
\hline $6-10$ & $17,8 \%$ & $9,4 \%$ & $9,4 \%$ \\
\hline $11-15$ & $16,6 \%$ & $16,4 \%$ & $-8,6 \%$ \\
\hline 15 und mehr & $23,7 \%$ & $17,2 \%$ & $41,0 \%$ \\
\hline
\end{tabular}

Quelle: Windolf 1999; Windolf/Beyer 2002

Es zeigt sich, daß das typische Muster der "Haus- und Kaminkarriere", in denen sich eine lange Bëtriebszugehörigkeit mit Spezialistenqualifikationen verbindet, trotz der Globalisierung nicht der Vergangenheit angehört.

Ein Blick wiederum nach Ostasien bestätigt diese Ergebriisse. Auch hier lassen sich die vieldiskutierten ,globalen Eliten" nicht finden. Zwar war in die letzten Jahren viel vom Ende des Clan-Kapitalismus oder, bezogen auf den chinesischen Wirtschaftsraum, der "old bamboo networks" die Rede, die im Zuge der Globalisierung und der Politiken von IWF und WTO an Grund verloren und Tür und Tor für das internationale Management geöffnet hätten. Aber de facto hat sich 2.B. sowohl in Japan als auch in Südkorea an der Rekrutierung der ökonomischen Eliten aus Clans, aus Familien, Schulkameradschaften und regionalen Netzen wenig geändert.

Südkorea ist dafuir auch deswegen ein sehr gutes Beispiel, weil die asiatische Finanzkrise mit ihren massiven Restrukturienungen der Großunternehmenslandschaft und der nicht unbedeutende Einfluß des IWF in Richtung einer weitreichenden Liberalisierung der südkoreanischen Ökonomie einen Wandel in Richtung eines internationalen oder noch besser: transnationalen Top-Managements hätte erwarten lassen können. 2000 sahen sich die noch bestehenden südkoreanischen Unternehmensgruppen von der Regierung veranlaßt, mehr als die Hälfte der Mitglieder des „,board of directors“ von 
außerhalb der Chaebol-Gruppe zu bestellen. Aber dies änderte nicht wirklich etwas an den Clanstrukturen der großformatigen Familienunternehmen. Die Macht ist in den südkoreanischen Untemehmensgruppen immer noch hoch konzentriert und die Südkoreas clanorientierte Elitenstruktur trotz neuer Elemente kaum aufgebrochen (vgl. dazu auch Hart-Landsberg/Burkett 2001: 419). Betrachtet man die Vorstandsvorsitzenden der zehn größten Unternehmensgruppen im Jahre 2003, so erkennt man, daß die Krise und die nachfolgende Restrukturierung an der familialen Reknitierung des Top-Managements nichts geandert hat.

Tab. 3: Die Top Ten-Unternehmensgruppen, ihre Präsidenten und ihre Beziehung zum Gründer

\begin{tabular}{|l|l|l|l|l|}
\hline $\begin{array}{l}\text { Chaebol } \\
\text { Ranking 2003 }\end{array}$ & $\begin{array}{l}\text { Gründer, } \\
\text { Präsidentschaft }\end{array}$ & $\begin{array}{l}\text { Präsident } \\
1996\end{array}$ & $\begin{array}{l}\text { Präsident } \\
1999\end{array}$ & $\begin{array}{l}\text { Präsident } \\
2003\end{array}$ \\
\hline Samsung & $1938-1987$ & Sohn & Sohn & Sohn \\
\hline LG & $1931-1969$ & Enkel & Enkel & Enkel \\
\hline Südkorea & $1953-1973$ & Neffe & Neffe & Neffe \\
\hline Hyundai A. & $1947-1987$ & Sohn & Sohn & Sohn, \\
\hline Lotte & seit 1967 & Gründer & Gründer & Gründer \\
\hline Shinsegae & $1930-1991$ & Tochter & Tochter & Tochter \\
\hline Hanwa & $1952-1981$ & Sohn & Sohn & Sohn \\
\hline Hanjin & seit 1945 & Gründer & Gründer & Sohn \\
\hline CJ & $1953-1993$ & Enkel & Enkel & Enkel \\
\hline Hyundai HI & $1947-1987$ & Sohn & Sohn & Sohn \\
\hline
\end{tabular}

Quelle: Pohlmann 2002, eigene Recherchen

Ebenso wie in Japan kann von einer Internationalisierung des Top-Managements auf diesen und auch den anderen Top-Managementpositionen nicht die Rede sein. Auch hier zeigt sich, daß die Entstehung einer globalen Elite, eines transnationalen Managements ein (Modernisierungs-)Mythos der Globalisierungsliteratur ist, der einer genaueren empirischen Prufung nicht stand zu halten vermag.

\section{Globalisierung und soziale Formen der Arbeits- und Lebensgestaltung:}

Abwärtsspiralen und regressive Entwicklung?

Bei den Antworten auf die Frage, wie sich die Globalisierung auf kulturelle und soziale Formen der Arbeits- und Lebensgestaltung auswirkt, folgen die Globalisierungstheorien ebenfalls Reflexionsimpulsen der (älteren) Modernisierungstheorie. Ihr gesellschaftskritischer Bezugspunkt wird in der Auflösung, Gleichschaltung oder Umformung de Lokalen und Traditionalen ebenso greifbar wie die angenommene Bedrohung lokaler und regionaler Errungenschaften. Der Globalisierung wird kultur- und sozialzerstörerische Kraft attestiert. Dort, wo sie neue Formen einführt, sich reue Denkweisen, Deutungsschemata, Werte und Normen global verbreiten, gingen alte kulturelle und soziale Traditionen zugrunde, verflachten oder würden gleichgeschaltet (vgl: dazu nur Cowen
2002; Lewis 2002 etc.). Aber sind jene Abwärtsspiralen im Wettlauf um Standortbedingungen, die eine Angleichung der Lebensbedingungen und sozialen Absicherungen auf dem kleinsten gemeinsamen Nenner bewirken - also zu einer Erosion wohlfahrtsorientierter Komponenten führen, tatsächlich die Regel? Und findet im Zuge fortschreitender Globalisierung eine Fortsetzung der Kolonialisierung von Lebenswelten mit noch weitreichenderen systemischen Mitteln statt?

Legt man eine allgemeinere Perspektive in der Frage der Erosion sozialer Standards durch die Globalisierung an, so kann man sehr klar erkennen, daß weltweit in generalisierender Weise von Abwärtsspiralen im Zuge forcierter Globalisierung kaum die Rede sein kann. Wiesenthal u.a. haben zurecht darauf hingewiesen, daß im internationalen Handel nicht generell die Verteilungsordnung eines Nullsummenspiels gelte und Wachstums- und Prosperitătsgewinne in den Industrieländern keineswegs zwangsläufig zu Einbußen bei den Schwellen- und Entwicklungsländern führen müssen und umgekehrt (vgl. Wiesenthal 1999: 515 ff.). Gerade die ostasiatischen Länder machten ja deutlich, daß Positivsummenspiele möglich sind und sie zeigten auch, daß eine Integration in die Globalökonomie nicht zwangsläufig mit einer weitreichenden Erosion sozialer Standards und einer Zunahme sozialer Ungleichheit erkauft werden muß. Denn Japan, Suldkorea und Taiwan gelten gerade im Aspekt der sozialen Ungleichheit in der internationalen Diskussion als "Vorzeigeökonomien", weil sie trotz forcierter Globalisierung und geringer wohlfahrtsstaatlichen Orientierungen die erkennbaren sozialen Verwerfungen gering halten konnten. Ebenso wie in Taiwan und Japan sind in Südkorea $z$.B. die Einkommensunterschiede zwischen der oberen und der unteren Einkommensgruppe vergleichsweise gering. Verglichen mit anderen Schwellen- und Entw icklungsländern hatten Südkorea - zusammen mit Taiwan - bereits in ihrer Wirtschaftswunderphase nach allen statistischen Angaben die geringsten Einkommensunterschiede. ${ }^{9}$ Diese Unterschiede haben sich nach den verfuggbaren Indikatoren in den Folge noch weiter verringert.

Ein Blick hinter diese Indikatoren zeigt zwar daß sich die Unterschiede zwischen Reich und Arm in Sudkorea, Taiwan und Japan insgesamt seit Ende der 80er Jahre ausgeweitet haben, während das soziale Sicherheitsnetz, so Adelman, im Vergleich zu den OECD-Ländern zunächst rudimentär geblieben ist (Adelman 1997: 529). Insbesondere sind prekäre Wohlfahrtslagen im Dienstleistungsbereich und im kleinbetrieblichen bzw. Selbständigensektor der Industrie häufig, da außerfamiliale soziale A bsicherungen weitgehend fehlen. Auch sind die Vermögen in schärferer Weise ungleich verteilt als die Einkommen (vgl. Choi/Kwon 1997: 568-572). Aber gerade deswegen wurden Fragen sozialer Wohlfahrt zu zentralen politischen Themen und wohlfahrtsstaatliche Programme in allen ostasiatischen Ländern sukzessive eingefuihrt. Von Abwärts-

9 Das Gehalt eines koreanischen Arbeiters lag 1988 gemessen an dem eines Angesteliten bei $63 \%$ und 1997 bej rund 79\%: Der in seiner Berechnung daran orientierte Indikator weist daher einen Ruckgang um 48 Indikatorpunkte von 1985 bis 1997 auf. Auch die immer noch vergleichsweise krassen geschlechtsspezifischen Unterschiede haben sich vermindert. Betrug das durchschnittliche Einkommen einer weiblichen Beschaftigten 1989 noch $51,4 \%$ des Einkommens eines mănnlichen Beschaftigten, so hat sicl dieser Prozentsatz bis 1997 auf $62,1 \%$ verbessert. Ebenso haben sich die Unterschiede im Einkom men nach Bildungsniveau abgemildert. Allein die Unterschiede zwischen Klein- und Großbetrieben habe deutlich zugenommen. Sie sind von I12 Indikatorpunkten 1985 auf 138 Indikatorpunkte gestiegen. 
spiralen kann hier also keine Rede sein. Auch diese Annahme scheint in ihrer generalisierten Form vielmehr ins Reich der (Modernisierungs-)Mythen der Globalisierungstheorie zu gehören.

In ähnlicher Weise hatte man in Deutschland von der Europäisierung eine Tendenz zum Abbau des sozialen Schutzniveaus, eine Art Sozialdumping erwartet. Politische wie politikwissenschaftliche Überlegungen sprachen noch vor wenigen Jahren für die Hypothese, daß die Initiativen zur Vollendung des europäischen Binnenmarktes soziales Dumping z.B. beim Arbeitsschutz nach sich ziehen und selbst die erreichten nationalen Schutzniveaus durch die Vereinheitlichung zur Disposition stellen würden. Zahlreiche Beiträge zur europäischen Sozialpolitik stützen diese These durch selektive Behandlung der verabschiedeten Richtlinien (vgl. Kowalsky 1999: 117). „Allen Erwartungen zum Trotz", so Kowalskis Analysen, „kam es im Gesundheits-, Arbeits-, Umwelt und Verbraucherschutz durch europäische Regulierungen (Arbeitsschutzrahmenrichtlinie 89/391, Maschinenrichtlinie 89/392, Bildschirmrichtlinie 90/270 etc.) weder zum sozialen Dumping noch zu einer Deregulierung und Absenkung des bisherigen Schutzniveaus, wie es insbesondere Gewerkschaften, Umweltverbände und Sozialpartner befürchteten und wie es aufgrund von politik- und sozialwissenschaftlichen Analysen der Entscheidungslogik im Europäischen Rat zunächst auch zu erwarten war" (Kowalsky 1999: 117). Im Gegenteil, so Kowalski weiter, durch rege Regulierungstätigkeit wurde ein hohes Schutzniveau, eine „Harmonisierung bei gleichzeitigem Fortschritt" zu erreichen versucht.

In bezug auf die Sozialhilfe richtete sich in Deutschland das kritische Augenmerk der Globalisierungstheorien auf eine zunehmende „Warenförmigkeit“ („Rekommodifizierung") der Sozialhilfe nach Maßgabe der Entwicklungen in den USA (vgl. dazu u.a. Leibfried 2001). Die These der zunehmenden "Warenförmigkeit" spricht einen Proze $\beta$ an, in dem die Sozialhilfeleistungen stärker an die Entäußerung von Arbeitskraft auf dem Arbeitsmarkt geknüpft werden. Leibfried kann nun zeigen, daß diese Diskurse der Umgestaltung der Sozialhilfe im Zuge der Globalisierung in den USA und Deutschland von sehr unterschiedlicher Bedeutung waren und es in beiden Fällen keine manifeste Auswirkung dieser Diskurse auf die Umgestaltung der Sozialhilfesysteme gab. Auf Basis von nach wie vor großen Strukturunterschieden sieht er zwar ähnliche Prozesse der „Rekommodifizierung“ der Sozialhilfe am Werke, aber die entgegengesetzte Kraft staatlicher Wohlfahrtsorientierungen bleibt für ihn in beiden Ländern in unterschiedlicher Weise erhalten. Keineswegs kann hier also von einem globalen Prozeß der Erosion von Sozialhilfe- und wohlfahrtsstaatlichen Standards die Rede sein, wie es viele Globalisierungstheorien nahelegen.

Dies gilt auch für die Frage einer kulturellen Erosion, einer zunehmenden Kolonialisierung und Gefährdung von Lebenswelten. Mückenberger machte hier im Globalisierungsschwerpunkt deutlich, daß die Kolonialisierungswirkungen global etablierter Systeme nur eine Seite der Medaille darstellen. Er zeigte auf, daß ihnen entgegengesetzt neuartige Vernetzungen entstanden sind, die stärker an die Alltagswelten der Bürger angekoppelt sind (Mückenberger 2001). Er spricht hier von einer neuen ,zivilgesellschaftlichen Struktur". Dieser neuen zivilgesellschäftlichen Struktur könne es gelingen, so die These, mittels kommunikativer Macht und Bürgerrechte auch verstreute, schwer organisierbare Bürgerinteressen zur Geltung zu bringen und so offensiv zu einer „Dekolonialisierung“ der Lebenswelt beizutragen.

Die Expansionskraft des globalen Kapitalismus zerstört hier keineswegs nur kulturelle Traditionen, sondern fordert auch immer wieder kulturell fundierte Identitătsbehauptungen in Abgrenzung dazu neu heraus. In der Auseinandersetzung mit Rationalisierungsmaßnahmen wird immer wieder auf alte und neue kulturelle Formen der gesellschaftlichen Organisation zurückgegriffen. Neu erfundene Traditionen im Hobsbawmschen Sinne (Hobsbawm/Ranger 1983) kommen ins Spiel. Nicht die umstandslose Durchsetzung einer Kolonialisierung durch das kapitalistische Weltsystem ist zu beobachten, welche die Kritiker des Neoliberalismus so sehr kritisieren, sondern ihre kulturell unterfuitterte Beschränkung.

Die Globalisierung, so könnte man die Forschungsbefunde zusammenfassen, läßt sich in ihren Auswirkungen für die Ausgestaltung der sozialen Absicherung und der alltäglichen Lebenswelt nicht einfach als zerstörerische Kraft beschreiben, die zur Dẹprivation, Erosion der sozialen Absicherung und Kolonialisierung der Lebenswelten führt, sondern sie regt gegenläufige Prozesse der Dekommodifizierung und Dekolonialisierung immer wieder an. Unklar bleibt jedoch, wie dies genau funktioniert. Die dahinter stehenden Prozesse und Mechanismen zu erfassen und theoretisch zu konzipieren, bleibt ein spannendes Aufgabenfeld der Globalisierungsforschung und -theorie.

\section{Schluß und Ausblick}

Die Globalisierungsforschung ermöglicht es heute, hinter die großangelegten Trendaussagen des Mainstreams der Globalisierungstheorien zurückzugehen und dessen Thesen auf ihre Erklärungsreichweite und Triftigkeit abzuklopfen. Das Ziel der vorangegangenen Ausführungen war es vor diesem Hintergrund, zu klären, ob sich die zentralen Annahmen der Globalisierungstheorien auf dem Prüfstand der empirischen Forschung bewähren oder ob diese nur mehr eingängige (Modernisierungs-)Mythen widerspiegeln.

Bezogen auf die Rolle der Staaten zeigte die Konfrontation mit dem entwicklungssoziologischen Diskurs und seinen Befunden, daß in generalisierender Weise von einer Erosion nationalstaatlicher Politiken im Zuge der Globalisierung nicht die Rede sein kann. Der Nationalstaat ist nicht nur nach wie vor die Form, die sich ein Gebiet gibt, um an Politik teilzunehmen. Sondern auch der globale Fluß des Kapitals reagiert auf institutionelle Variationen, die maßgeblich durch nationalstaatliche Politiken bestimmt sind. Sie geben der Weltwirtschaft ihre konzentrische und triadische Struktur und bestimmen die Entwicklungschancen einzelner Ökonomien in erheblichem Maße mit, wie am Beispiel Ostasiens einfach erkennbar. Zwar hat sich die Bedeutung nationalstaatlicher Souveränität im Zuge der Globalisierung verändert, aber die Instrumente nationalstaatlicher Politik sind nach den entwicklungssoziologischen Befunden weder unwirksam geworden noch ist davon aus̊zugehen,- daß die nationalstaatliche Verfaßtheit keine entscheidende Rolle mehr spielt. Hinter dieser Annahme der Globalisierungstheorien steckt vielmehr eine Mythologisierung der globalen Ökonomie als Mo- 
tor für eine Entwicklung globaler gesellschaftlicher Formen, die ältere nationalstaatliche obsolet werden lasse. Der Gedanke gleicht jenem älterer Modernisierungstheorien: Neue moderne Formen lösen traditionale ab und ersetzen sie. Die Möglichkeit ihrer gleichzeitigen Präsenz ist in den Entwicklungsannahmen des Mainstreams der Globalisierungstheorien nicht mitgedacht.

Ein genauerer Blick auf die empirischen Befunde der Globalisierungsforschung zeigt weiter an, daß das (im Mainstream der Globalisierungstheorien implizit dominierende) Credo der neo-liberalen Theorie, daß nur Regionen prosperieren, die sich dem Weltmarkt gänzlich öffnen und sich global integrieren, keine generelle Geltung beanspruchen kann. Die avisierten Vorteile einer Delokalisierung regionaler Produktion stellen sich keineswegs generell und zwangsläufig ein. Dies gilt um so mehr, wenn globale Unternehmen sich ansiedeln. Synergieeffekte für die regionalen Räume ließen sich in einigen Fällen gar nicht feststellen; in anderen waren die Auswirkungen für die Regionen desaströs. Und in Ostasien stehen mit Japan, Südkorea und Taiwan ausgerechnet Ökonomien an der Spitze, die eine Ansiedlung globaler Unternehmen industriepolitisch vermieden haben. Die Gleichung, globale Integration = regionale Prosperität' geht also in mehrfacher Hinsicht empirisch nicht auf, auch wenn dies durch neo-liberale Theorien und durch den IWF immer wieder proklamiert wird.

Auch die Entstehung einer globalen Elite, eines transnationalen Managements ist nach den vorliegenden empirischen Befunden ins Reich der Mythen der Managementliteratur und einiger Globalisierungstheorien zu verweisen. Mitnichten kann von einer Weltkultur des Managements die Rede sein, noch dienen diese als Träger einer Modernisierung sich entwickelnder Ökonomien, wie in manchen Globalisierungstheorien nahegelegt.

Viele Globalisierungstheorien überschätzen also die Bedeutung transnationaler Unternehmen (und ihres Managements), ja schätzen sie in ihrer Schubkraft für die Modernisierung zurückgebliebener Ökonomien falsch ein. Die im entwicklungssoziologischen Diskurs gut verankerte Perspektive, daß sich mit ihnen gerade keine Steigerung formaler Rationalitäten des Wirtschaftens verbindet, geht im Mainstream der Globalisierungstheorien zumeist verloren, auch wenn die Globalisierungsforschung dafür zahlreiche empirische Befunde zur Verfügung hält. Erst in der Dramatisierung der sozialen Folgen, also (im Weberschen Sinne) einer materialen Irrationalität der ,Transnationalisierung" durch transnationale Unternehmen, setzt der Chorus der Globalisierungstheorien wieder ein - und teilt damit wiederum das Rationalitätsverdikt und die Fortschrittsassoziationen älterer Modernisierungstheorien.

Doch auch jene vom Mainstream der Globalisierungstheorien gerne beklagten Abwärtsspiralen im Wettlauf um Standortbedingungen, die Zerstörung oder Gleichschaltung kultureller und sozialer Traditionen lassen sich auf dem Prüfstand der empirischen Forschung nicht einfach bestätigen. Zwar gibt es z.B. in bezug auf die Knüpfung der Sozialhilfe an die Entäußerung von Arbeitskraft weltweit ähnliche Rationalisierungsimpulse; aber sie setzen sich weder bruchlos noch pfadunabhängig durch. Viel eher bleiben in unterschiedlicher Weise staatliche Wohlfahrtseinrichtungen erhalten, denen es gelingt, ihre institutionelle Stoßkraft (in dekommodifizierender Weise) der Ökonomisierung und „Vermarktlichung“ der Sozialhilfe entgegenzusetzen. Bereits aus dieser Entgegensetzung ergeben sich unterschiedliche Rationalisierungspfade, welche die Vorstellung, Globalisierung schlage alles mit Ähnlichkeit, ad absurdum führen. Dasselbe Muster decken die hier vorliegenden Forschungsbefunde in der Frage der Kolonialisierung auf. Die Belebung zivilgesellschaftlichen Engagements und die Entfaltung neuer zivilgesellschaftlicher Strukturen ist auch ein sozialer Effekt von Globalisierung - einer Globalisierung, die gegenläufige Prozesse der Dekommodifizierung und Dekolonialisierung immer wieder anzuregen scheint. Soziale Abwärtsspiralen jedenfalls sind kein genereller Effekt von Globalisierung und Standortkonkurrenz, wie sich an den Entwicklungstendenzen in Ostasien, aber auch in Europa einfach ablesen läßt. Auch hier weisen die Befunde der Entwicklungssoziologie in eine andere Richtung als die Annahmen im Mainstream der Globalisierungstheorien, die auch in diesem Aspekt das Argumentationsmuster älterer Modernisierungstheorien übernehmen.

Insgesamt stellt sich also heraus, daß der Mainstream der Globalisierungstheorien von der Umstellung ihres Beobachtungsschemas solange nicht profitieren kann, wie Globalisierung und Modernisierung in seinen Annahmen kurzgeschaltet werden und so vermeintliche Aufklärung über die Effekte der Globalisierung in Mythologie umschlägt. Seine grundlegenden Annahmen haben sich in ihrer generalisierten Form auf dem Prüfstand der empirischen Befunde der Entwicklungssoziologie und der Globalisierungsforschung nicht bewähren können. Viele Globalisierungstheorien treten mit diesen Annahmen vielmehr das unreflektierte Erbe älterer Modernisierungstheorien an; das sich schon in der Vergangenheit als nur begrenzt tauglich für die Beschreibung und Beobachtung von Gesellschaften und ihrer Entwicklung erwiesen hat. Die Vorteile, welche die Globalisierungstheorien durch den Wechsel ihrer Leitunterscheidung gegenüber Modernsierungstheorien haben, bleiben auf diese Weise jedenfalls ungenutzt.

\section{Literatur}

Adelman, Irma (1997): Social Development in Korea, 1953-1993, in: Cha, Dong-Se et al. (eds.): The Korean Economy 1945-1995: Performance and Vision for the 21 $1^{\text {si }}$ Century, Seoul: KDI (509-540) Albrow, Martin (1998): Auf dem Weg zu einer globalen Gesellschaft? in: Beck, Ulrich (ed.): Perspektiven der Weltgesellschaft, Frankfurt/M.: Suhrkamp

Amsden, Alice (1989): Asia's Next Giant. South Korea and Late Industrialization, New York, Oxford: Oxford University Press

Amsden, Alice (1990): Third World Industrialization: Global Fordism or a New Model?, New Left Review, Vol. 182: 5-31

Beck. Ulrich (1997): Was ist Globalisierung? Franfurt/M.: Suhrkamp

Beck, Ulich (1997): Was ist Globaliceng? Franfing : Sul kumulative Prozesse zirkulärer Verursachung?, Abschlußbericht DFG, Erlangen

Camilleri, Jo Pozh A. Jim Falk (1992): The End, Abschere ting World, Vermont: Edward Elgar

Castells, Manul (1992): Four Asian Tigers with a Dragon Head. A Comparative Analysis of the State Castells, Manuel (1992). Pour Asian Tigers with a Dragon Head. A Comparative Analysis of the State, Economy, and Society in the Asian Pacific Rim, in: Appelbaum, Richard P., Henderson, Jeffrey (eds.):
States and Development in the Asian Pacific Rim, Newbury Parc et al.: Sage (176-198)

- Choi, Kwang, Soonwon Kwon (1997): Social Welfare and Distribution Policies, in: Cha, Dong-Se et al. (eds.): The Korean Economy 1945-1995: Performance and Vision for the 21 $1^{\text {t }}$ Century, Seoul: KDI (541585) 
Cowen, Tyler (2002): Creative Destruction. How Globalization is Changing the World's Cultures, Priceton, Princeton University Press

Cumings, Bruce (1989): Ursprünge und Entwicklung der politischen Ökonomie in Nordostasien: Industriesektoren, Produktzyklen und politische Konsequenzen, in: Menzel, Ulrich (ed.): Im Schatten des Siegers: Japan, Bd. 4: Weltwirtschaft und Weltpolitik, Frankfurt/M.: Suhrkamp (87-145)

Cumings, Bruce (1997): Korea's Place in the Sun. A Modern History, New York, London: Norton \& Com-

pany
Deyo, Frederic C. (1989): Beneath the Miracle. Labor Subordination in the New Asian Industrialism, Berkeley et al.: University of California Press

Feldhoff, Jürgen, Philipp Hessinger (2001): Industriedistrikte als industriepolitisches Handlungsfeld, Abschlußbericht DFG, Erlangen

Gerschenkron, Alexander (1962/66): Economic Backwardness in Historical Perspective, in: Landes, David (ed.): The Rise of Capitalism, New York: MacMillan

Hahm, Sung Deuk, Christopher L. Plein (1997): After Development. The Transformation of the Korean Presidency and Bureaucracy, Washington: Georgetown University Press

Hamilton, Gary G., Nicole Woolsey Biggart (1988): Market, Culture and Authority: A Comparative Analysis of Management and Organization in the Far East, American Journal of Sociology, Vol. 94, Supplement: $52-94$

Hartmann, Michael, Johannes Kopp (2001): Elitenselektion durch Bildung oder durch Herkunft? Der Zugang zu Fuhrungspositionen in der deutschen Wirtschaft, KZfSS, Vol. 53, No. 3: 436-466

Hartmann, Michael (2002): Der Mythos von den Leistungseliten. Spitzenkarrieren und soziale Herkunft in Wirtschaft, Politik, Justiz und Wissenschaft, Frankfurt a. M.: Campus

Hart-Landsberg, Martin, Paul Burkett (2001): Economic Crisis and Restructuring in South Korea. Beyond the Free Market-Statist Debate, Critical Asia Studies, Vol. 33, No. 3: 403-430

Henderson, Jeffrey, Richard B. Appelbaum (1992): Situating the State in the East Asian Development Process, in: Appelbaum, Richard P., Henderson, Jeffrey (eds.): States and Development in the Asian Pacific Rim, Newbury Parc et al.: Sage (1-31)

Henderson, Jeffrey (1993): Against the Economic Orthodoxy: On the Making of the East Asian Miracle Economy and Society, Vol. 22, No. 2: 200-217

Heinze, Rolf G., Heiner Minssen (2001): Regionalisierungstendenzen in Wirtschaft und Politik am Beispiel der Umstrukturierung von Opel in Bochum und Opel in Eisenach, Abschlußbericht DFG, Erlangen Hirschman, Albert (1958): The Strategy of Economic Development, New Haven: Yale University Press Hobsbawm, Eric, Terence Ranger (eds.) (1983): The Invention of Tradition, Cambridge: University Press Jwa, Sung-Hee; Huh Chan Guk (1998): Korea's 1997 Currency Crisis: Causes and Implications, Korea Journal, Vol. 38, No. 2: 5-33

Johnson, Chalmers (1982): MITI and the Japanese Miracle, Stanford: University Press

Kowalski, Wolfgang (1999): Europäische Sozialpolitik. Ausgangsbedingungen Antriebskrafte und Entwicklungspotentiale. Opladen: Leske und Budrich

(2) Erlangen

Global Trends in the $21^{\text {st }}$ Century, Yarmouth: Intercultural Press

Luhmann, Niklas (1992) Beobachtungen der Moderne, Opladen: Westdeutscher Verlag

Luhmann, Niklas (1997): Die Gesellschaft der Gesellschaft, Frankfurt/M.: Suhrkamp

Niklas (1998): Der Staat des politischen Systems, in: Beck, Ulrich (ed.): Perspektiven der Weltgesellschaft, Frankfurt/M.: Suhrkamp

Mandel, Ernest (1972): Der Spătkapitalismus, Frankfurt/M.: Suhrkamp

Mückenberger, Ulrich (2001): Zivilgesellschaft und disperse Interessen, Abschlußbericht DFG, Erlangen Narr, Wolf-Dieter, Alexander Schubeit (1994): Weltókonomie. Die Misere der Politik, Frankfurt/M.: Suhrkamp

Ohmae, Kenichi (1995): The End of the Nation State. The Rise of Regional Economies, New York et al. Free Press

Pohlmann, Markus (2002) „Der Kapitalismus in Ostasien. Súdkoreas und Tajwans Wege ins Zentrum der Weltwirtschaft", Münster: Westfalisches Dampfboot

Pohlmann, Markus (2004): Die Entwicklung des Kapitalismus î̉ Ostasien und die Lehren aus der asiatischen Finanzkrise, Eeviathan, №.3

Pre

Reich, Robert (1993): Die neue Weltwirtschaft Das Ende der nationalen Ökonomie, Frankfurt/M: Fischer Ritzer, George (2000): The McDonaldization of Society, 3. Aufl., Pine Forge: Sage
Schmidt, Gert (1999): „Globalisierung“- oder: Der gesellschaftliche Diskurs zur Sorge ums Weitermachen am Ende des 20. Jahrhunderts, in: Schmidt, Gert, Rainer Trincek (eds.): Globalisierung. Ökonomische und soziale Herausforderungen am Ende des zwanzigsten Jahrhunderts, Sonderband 13 der Sozialen Welt, Baden-Baden: Nomos: 11-28

Stichweh, Rudolf (2000): Die Weltgesellschaft. Soziologische Analysen, Frankfurt/M.: Suhrkamp

Trimberger, Ellen Kay (1979): World Systems Analysis: The Problem of Unequal Development, Theory and Society, Vol. 8: 127-137

Wade, Robert (1990): Governing the Market. Economic Theory and the Role of Government in East Asian Industrialization, Princeton: Princeton University Press

Wallerstein, Immanuel (1974): The Modern World-System (Vol 1), New York: Academic Press [deutsch: (1986): Das modeme Weltsystem - Die Anfange kapitalistischer Landwirtschaft und die europäische Weltokonomie im 16. Jahrhundert, Frankfurt/M: Syndikat]

Wallerstein, Immanuel (1979): The Capitalist World-Economy, Cambridge et al.: Cambridge University

Press
Wallerstein, Immanuel (1991) [1995]: Unthinking Social Science. The Limits of Nineteenth-Century Paradigms, Cambridge: Polity Press [deutsch: (1995): Die Sozialwissenschaft 'kaputtdenken'. Die Grenzen der Pardigmen des 19. Jahrhunderts, Weinheim: Beltz. Athenaum

Weede Erich (1990): Wirtschaft, Staat und Gesellschaft: Zur Soziologie der kapitalistischen Marktwirtschaft und der Demoksatie, Tubingen: Mohr

Wiesenthal, H. Globalisierung als Epochenbruch - Maximaldimensionen eines Nullsummenspiels, in Schmidt, Gert, Rainer Trincek (Hrsg.): Globalisierung. Ökonomische und soziale Herausforderungen am Ende des zwanzigsten Jahrhunderts, Baden-Baden: Nomos (503-533)

Windolf, Paul, Jürgen Beyer (1995): Kooperativer Kapitalismus. Unternehmensverflechtungen im internationalen Vergleich. Kölner Zeitschrift fur Soziologie und Sozialpsychologie, 47, 1-27

Windolf Paul, Jürgen Beyer (2002): Corporate Networks in Britain and Germany, in: British Journal of Sociology, 47/2, 205-231 